\title{
The Field Modelling of Fire in an Air-Supported Structure
}

\author{
K. A. PERICLEOUS and D. R. E. WORTHINGTON \\ CHAM Limited \\ 40 High Street \\ Wimbledon, London SW19 5AU, UK
}

G. $\operatorname{cox}$

Fire Research Station

Borehamwood

Herts, WD6 2BL, UK

\section{ABSTRACT}

The field modelling technique for predicting the temperature distribution and smoke movement in enclosures containing a fire source is validated against experiments carried out in a fully instrumented sports building covered by an air supported dome. The building is oval in plan and the dome has an ellipsoidal shape.

A $2 \mathrm{MW}$ methanol pool fire located centrally on the floor of the building was used to obtain detailed measurements of temperature at a number of locations. The mathematical model simulates the transient problem in three dimensions using two different finite volume grids. The first grid is a polar cylindrical one with cells partially blocked to simulate features not coincident with grid lines. The second uses a non-orthogonal grid which follows closely the contours of the building. Results are obtained for pre-fire, fire and post-fire conditions and the two grid solutions are compared with experiments.

Qualitative agreement is good throughout and trends are correctly simulated. Quantitative agreement is also good in all areas except in common with earlier studies in the immediate vicinity of the fire source. The body fitted grid solution predicts correctly the lack of stratification due to strong convection along the ceiling.

Keywords: Mathematical model, smoke, body-fitted coordinates, fire

\section{INTRODUCTION}

The mathematical field modelling of fire has now reached the stage where two distinct branches of further development are taking place. On the one hand smoke movement models, already reasonably well validated at least in all except the very largest of buildings [1], are being applied to practical problems for design purposes. This is leading to refinements of the models in the treatment of the physical phenomena involved (eg. the prediction of carbon monoxide [2]) and in the complexity of the geometries being tackled [3]. There are also developments of user-friendly tools for easier use by the non-expert and for incorporation into 'systematic' fire models which fuse developments in the modelling of human escape, life threat and structural response with those of the gas phase.

On the other hand, and building on these capabilities, is the development of 'fire spread' models where the incorporation of more realistic radiation models [4] can be coupled with the development of semi-empirical treatments of material flame spread [5].

\section{c. Crown Copyright 1987}


This paper deals with the further development and validation of smoke movement models. The vehicle for the study is an experimental air supported dome in which Nakamura and his colleagues at the Shimizu Construction Company [6] conducted a number of fire tests. The experimental facility has major dimensions $34 \mathrm{~m} \times 28 \mathrm{~m} \times$ $11.6 \mathrm{~m}$ in height, being a one sixth scale model of a proposed sports stadium $(200 \mathrm{~m} x$ $200 \mathrm{~m} \times 70 \mathrm{~m}$ ). Even in its one sixth scale this building represents, as far as the authors are aware, the largest single volume used for field model validation. In addition to the value of the study as further model validation at large scale, it also offers because of the shape of the roof, an opportunity for testing and developing a body-fitted-coordinate version of the field model JASMINE.

The details of JASMINE have been described in detail elsewhere $[1,7,8]$. It is based on the general fluid dynamics package PHOENICS [9] as the main equation solver.

The work described here represents the development of a second version of the model to be known as JASMINE II which is based on the latest release of PHOENICS with improved numerical features and possesses a generalised non-orthogonal coordinate scheme.

\section{PHYSICAL MODEL SIMULATED}

The experimental building under consideration, constructed by Shimizu Construction Ltd, of Tokyo, Japan is an oval sports stadium with an air supported roof. It was designed as a $1 / 6$ th scale model of a proposed full size stadium which would hold a capacity crowd of 60,000 people. The dimensions of the model are, $11.6 \mathrm{~m}$ in height at the dome apex and $34 \mathrm{~m}$ by $28 \mathrm{~m}$ in plan. It was fully instrumented, in particular for the measurement of temperatures. Figure 1 shows a plan and side elevation together with the positions of the thermocouple rakes.

The dome was held aloft by an overpressure of $25 \mathrm{~mm}$ water gauge. This pressure was supplied by compressors located in two internal "sheds" shown in the figure. Air at a controlled temperature was supplied through a series of siots in a ring located around the periphery of the stadium, at a height of $7 \mathrm{~m}$. Air supply was computer controlled and divided into four quadrants. A continuous supply was necessary to compensate for air losses through the dome and walls. The dome material was constructed for lightness using an $.8 \mathrm{~mm}$ glass fibre skin. Air gaps exist along the various sections of the roof but not through the skin.

Nineteen fire tests were conducted with a fire source placed at various locations on the floor. In the present calculation one of these with a centrally located fire source is modelled. The fire source comprised four $1 \mathrm{~m}^{2}$ square trays of methanol, arranged in a square of $2.29 \mathrm{~m}$. The fire duration was 11 minutes with an estimated peak heat release rate of $2.2 \mathrm{MW}$. Measurements were taken before, during and after the fire had been extinguished and these were used in the comparison presented here.

One further point to mention is that, throughout the experiment two opposing quadrants only supplied the air for the dome. Table 1 shows the external air supply temperature and mass flow rates and also the amount of air recirculated for temperature control.

\section{Table 1: Air Conditioning Settings}

\begin{tabular}{ccccc}
\hline Quadrant & Total Massflow & Supply Temp & Recycle Rate & Velocity \\
2 & $3.023 \mathrm{~kg} / \mathrm{s}$ & $31.9 \mathrm{~K}$ & $2.124 \mathrm{~kg} / \mathrm{s}$ & $0.696 \mathrm{~m} / \mathrm{s}$ \\
4 & $2.312 \mathrm{~kg} / \mathrm{s}$ & $13.3 \mathrm{~K}$ & $1.625 \mathrm{~kg} / \mathrm{s}$ & $0.5 \mathrm{~m} / \mathrm{s}$ \\
\hline
\end{tabular}


As the two quadrants blow air at different temperatures, they induce recirculation in the flow. This recirculation is important as it dictates the way smoke spreads during the first few minutes of the fire, an important consideration for smoke detection device placement. For this reason, this pre-fire condition was calculated first.

\section{THE MATHEMATICAL MODEL}

The mathematical method used in this investigation is essentially the same as the one used in a previous investigation by the present research team and will not be discussed in detail here. The interested reader should consult References $[7,8,10]$ for full details. It is sufficient to say here that equations for the conservation of mass, momentum, enthalpy and fuel mixture fraction, to desribe the concentration of smoke, are solved throughout the calculation domain either on a cylindrical polar or a body-fitted coordinate grid. The two equation $k-\epsilon$ model is used to describe turbulent mixing. One important addition is the treatment of the effects of buoyancy on the source term $S_{\epsilon}$ in the $\epsilon$ equation. The modification recommended by Bos et al [10] accounts for the varying direction of the ceiling jet following the contours of a curved roof relative to the gravitational vector.

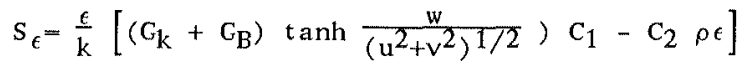

where $G_{k}$ and $G_{B}$ are the generation terms for turbulent kinetic energy due to shear and buoyancy respectively which take their usual form [11].

A four flux model was used to describe radiant heat exchange between adjacent cells. The four fluxes used were aligned along the longitudinal (vertical) and radial ordinates. The treatment of the body-fitted-coordinate grid has been described by Markatos et al [12].

\section{Boundary Conditions}

These are entered in the form of additional source terms to represent friction and heat transfer at the boundaries due to convection, conduction and radiation. For friction, $\log$-law wall functions are used to act as sinks of momentum in the appropriate velocity direction.

Similarly for the convective heat transfer at the walls the so called Reynolds analogy between momentum and heat transfer is employed. This requires the wall temperatures which are calculated from a balance at the boundary

$-U_{e m}\left(T_{m}-T_{e}\right)+U_{m p}\left(T_{p}-T_{m}\right)=\rho \frac{C p_{w} B\left(T_{m}-T_{m} *\right)}{\Delta t}$

where the subscripts $e, m$ and $p$ refer to external, wall mean and internal temperature respectively and the heat transfer coefficients are given by,

$\mathrm{U}_{\mathrm{mp}}=\frac{1}{\frac{1}{\mathrm{~h}_{\mathrm{i}}}+\frac{\mathrm{B}}{2 \mathrm{k}_{\mathrm{c}}}} \quad$ and $\quad \mathrm{U}_{\mathrm{em}}=\frac{1}{\frac{1}{\mathrm{~h}_{\mathrm{o}}}+\frac{\mathrm{B}}{2 \mathrm{k}_{\mathrm{c}}}}$

$B$ is the wall thickness, $k_{c}$ the conductivity and $h$ the convective heat transfer coefficient. $\mathrm{T}_{\mathrm{m}}{ }^{*}$ is the "old" wall mean temperature calculated at the previous time step. A minimum convective wall heat transfer limit is set at the natural convection limit given by

$$
\mathrm{Nu}=0.13 \mathrm{Ra}^{1 / 3}
$$


where the temperature gradient would lead to natural convection at the boundary. At the ceiling the combined convective and radiative heat transfer coefficient $h_{i}$ was prescribed as in [1]. The boundary condition for the radiation fluxes takes into account the wall emissivity and temperature.

The fire is entered as a heat source per unit area and also as a source of mass. This mass also enters the conservation equation representing the concentration of burned fuel in the building. Finally, the mass and momentum entering the two active air conditioning quadrants is introduced, and air leakage resistances adjusted to achieve the required over-pressure in the dome.

\section{The Solution Method}

The resulting set of partial differential equations was solved numerically by the JASMINE/PHOENICS code, with JASMINE being the problem specifying element and PHOENICS comprising the equation solver. Details relating to the solution and formulation of the finite-domain equations and the algorithms used are given in [7].

The finite domain representation of the problem is shown in Figure 2 in cylindrical polar and body-fitted coordinate form. A total of 2860 computational cells is used in the polar grid case compared to 1200 in the body fitted coordinate case. However, in the polar grid case about $15 \%$ of the cells are unused as they lie outside the calculation domain and are "blocked off". The number of cells used, was chosen as a compromise between accuracy and economy and therefore no claim of grid independence is made. The comparison with experiments was deemed to be satisfactory nevertheless and due to uncertainties in experimental measurements it was felt that further refinement at this stage in terms of validation would be unnecessary.

\section{RESULTS OF CALCULATION}

\section{Calculations Performed}

The calculation was split into three parts:

(i) a pre-fire steady state calculation;

(ii) a transient calculation for the duration of the fire; and,

(iii) a post-fire transient calculation following the burn-out of the fuel.

Each one of the transient cases involved a number of time-steps and the result of each timestep was stored in the form of a field of velocities, pressure, temperature and fuel concentration and then plotted using a post-processor. The amount of results so produced is very large and in the space available only a small sample can be presented, in condensed form.

In the main fire transient (ii), the heat source was modelled by a linear growth over the first 90 seconds to the maximum constant level of rate of heat release of $2.2 \mathrm{MW}$ over the next 5.5 minutes and a linear decay from this level to zero for a further four minutes as the fuel becomes exhausted. The numerical timesteps were chosen to ensure timestep independence in the initial critical stage and to resolve the spreading of the combustion products under the ceiling. These varied between 2 seconds and 10 seconds in the initial increasing ramp, to 30 seconds for the steady and decaying phases of the fire.

Results are presented for velocity vectors and contours of temperature and fuel mixture fraction in Figures 3,4 and 5. In Figures 6 and 7 for direct comparison with the experimental data, temperatures on thermocouple rake 3 are displayed. 
The pre-fire condition is shown in Figure 3, it shows a horizontal section below the roof and also a vertical section through a plane crossing the top of the control sheds. The dominant feature of the flow is a large scale recirculation caused by the air-conditioning inlets which supply air at different temperatures. hence, air rises in the vicinity of quadrant 2 while it falls near quadrant 4. Maximum velocities lie close to the roof and reach $0.5 \mathrm{~m} / \mathrm{s}$. Due to this circulation the temperature overall is quite uniform at a mean value of $17^{\circ} \mathrm{C}$.

Fire and Post-Fire Transient Results

The development of the transient calculation for the fire's duration is depicted in two different ways. Figure 4 shows a series of sections through the centre of the building viewed from the east. These sectional views show velocities and temperature contours at selected time intervals throughout the fire and just into the post-fire condition. The spread of combustion products just under the ceiling is shown during the initial growth phase of the fire in Figure 5 together with velocity vectors.

The initial prefire circulation in the stadium leads to an asymmetry during the early stages of the fire. By about two minutes this is largely overcome and the temperature. contours outside of the rising plume become horizontal. As the fire diminishes in rate of heat release over the period from seven to eleven minutes the plume thickens as the buoyancy reduces. At ten minutes the plume is very wide and there are large scale circulations on either side of it. By 11.5 minutes the fire is out, the buoyant plume has disappeared and an inverse plume appears at the roof due to ambient cooling. The temperature throughout the volume is fairly uniform.

\section{Smoke Spread}

Figure 5 shows the spread of combustion products and the velocities below the roof fabric. The flow field initially is that of the pre-fire condition. After 14 seconds the plume first touches the ceiling and as time proceeds, the flow field changes from a lateral flow to a radial impact jet. The initial condition leads to asymmetric contours which persist over most of the first two minutes. The values shown on the contours are for the fuel mixture fraction, which can be related to product concentration by the use of the reaction equation. At 14 seconds, the 0.0005 contour reaches the roof while at 70 seconds it reaches the stadium wall, a mean distance of $17 \mathrm{~m}$. A rate of spread of the products of combustion of about $0.3 \mathrm{~m} / \mathrm{s}$ is therefore indicated.

At two minutes from ignition (not shown) smoke concentrations are up on this at the centre of the roof by a factor of 5 .

\section{COMPARISON WITH EXPERIMENT}

\section{Scope of Experimental Data}

Five vertical arrays of thermocouples were placed in various positions in the stadium as depicted in Figure 1. Readings were then logged on a micro-computer every 30 seconds. In addition to temperatures, a velocity probe was placed close to the stadium wall which recorded a maximum vertical velocity of the order of $0.2 \mathrm{~m} / \mathrm{s}$. Smoke was released into the normally clean-burning fire plume and observed on a video camera to provide a qualitative indication of smoke spread.

The thermocouple readings are the most substantial and reliable form of data from the transient test and were therefore used here to compare with predictions. 
Figure 6 shows predicted and experimental temperature behaviour during the fire transient for thermocouple array positions 1 and 3 for the body-fitted grid. The dotted lines indicate the envelope of experimental values on the rake, and the solid lines the prediction at various heights.

The uppermost trace lies closest to the fire and demonstrates clearly the ramp-up, constant burning rate and ramp-down sections. Although the predictions match well the experiments, the broad agreement near the source is fortuitous, previous studies, eg. $[1,10]$ have shown that although far field predictions are reliable, accurate temperature fields close to the source cannot be expected without a considerably refined grid at that location. However, away from the source the agreement is reasonably good.

Figure 7 shows the temperature rise with height measurements and predictions for both grids used, at rake $3,11 \mathrm{~m}$ from the fire. Both grids show reasonable agreement with the data except near the ceiling where the bfc grid seems far superior. The cylindrical polar grid shows an apparently well mixed ceiling layer not supported by the experiment nor the bfc calculation. Clearly the bfc grid better represents the shape of the ceiling so perhaps this result is not surprising.

Finally as a summary of the practical implication of the experimental and numerical study a plot of the height of the $10 \mathrm{degC}$ temperature rise contour with time is shown in Figure 8 for rakes 3,4 and 5 . The agreement at head height (say $2 \mathrm{~m}$ ) is reasonably good except for the wall station where the model slightly underpredicts the time for this temperature contour to arrive.

\section{CONVERGENCE AND COMPUTING REQUIREMENTS}

\section{Convergence}

The coupling of momentum and energy fields through density, combined with the effects of buoyancy leads to non linear behaviour typical to high Rayleigh number situations. Hence, inertial under-relaxation was necessary for convergence coupled with a large number of iterative sweeps per timestep. Similar relaxation was used for the polar and bfe grids. Typically 100 sweeps were sufficient for convergence with cumulative volumetric errors being $1.5 \mathrm{~m}^{3} / \mathrm{s}$ compared to a domain volume of $8000 \mathrm{~m}^{3}$.

\section{Computing Requirements}

Computing requirements on a Concurrent 3280 super mini computer are summarised below in Table 2 for cylindrical-polar and body-fitted grid simulations.

Table 2: Computing Requirements

\begin{tabular}{lll}
\multicolumn{1}{c}{ Grid } & \multicolumn{1}{c}{ Polar } & \multicolumn{1}{c}{ BFC } \\
\hline Program Size & 1.6 Mbytes & 1.6 Mbytes \\
Ce11 Number & 2860 & 1200 \\
Time for Transient & $71 / 2$ hrs & 5 hrs \\
Time/(Sweep, Ce11, & & \\
Variable, Timestep) & $3.78 \times 10^{-4} \mathrm{~s}^{-1}$ & $7.5 \times 10^{-4} \mathrm{~s}^{-1}$ \\
\hline
\end{tabular}




\section{CONCLUSIONS}

Reasonable validation of these computational fluid dynamic field models has already been demonstrated for the treatment of smoke movement problems.

The study conducted here adds a large volume building to those already satisfactorily validated and in addition has provided a very important opportunity to develop the body-fitted-coordinate capability to the model JASMINE. This is an important development for the treatment of many building designs being proposed in modern architectures.

In common with earlier studies, comparisons with the data provided by the Shimizu Corporation show good agreement remote from the fire source but poor agreement in its immediate vicinity. This is to be expected with the coarse grid prescription of the source.

The calculations demonstrate that in a building of this type the familiar well mixed hot layer bath does not form, mainly due to a strong radial jet emanating from the point of plume impact on the ceiling.

The body fitted grid represents the resulting ceiling boundary layers correctly and hence yields the best result without sacrifice in economy.

The number of time steps and cells used in this work was kept at economical levels to allow many runs during model development. It is not therefore expected that the results are either grid independent or timestep independent. Good agreement for all practical purposes has nevertheless been achieved, adding confidence in the usefulness of the method in fire predictions to this scale of enclosure.

\section{ACKNOWLEDGMENT}

The authors wish to thank Mr Hiroyuki Nakamura and the Institute of Technology, Shimizu Construction Company for providing freely the test data used in this simulation. The paper forms part of the work of the Fire Research Station, Building Research Establishment Department of the Environment, UK. It is contributed by permission of the Director of the Building Research Establishment.

\section{REFERENCES}

1. Cox G, Kumar S and Markatos N C 'Some Field Model Validation Studies' First International Symposium on Fire Safety Science, p159, Hemisphere, 1986.

2. Moss J B, Cox G and Kumar $S$ 'Laminar Flamelet Models of Fire - the Prospect' Fundamental Aspects of Polymar Flamability, p53, Institute of Physics, 1987.

3. Galea $E R$ and Markatos $N$ C 'A Review of Mathematical Modelling of Aircraft Cabin Fires' Appl. Math. Modelling, 11, 162, 1987.

4. Shah N G 'New Method of Computation of Radiant Heat Transfer in Combustion Chambers' PhD Thesis, Imperial College, London, 1979.

5. Quintiere J G, 'A Simplified Theory for Generalizing Results from a Radiant Panel Rate of Flame Spread Apparatus, Fire and Materials' 5, 52, 1981 
6. H Nakamura 'Fire Behaviour Tests in an Air-Supported Structure' private communication, 1986.

7. Markatos N C and Cox G 'Hydrodynamics and Heat Transfer in Enclosures Containing a Fire Source' Physicochemical Hydrodynamics, 5, 53, 1984.

8. Markatos N C and Pericleous K A 'An Investigation of Three-Dimensional Fires in Enclosurès' Fire Dynamics and Heat Transfer, p115, ASME,1983

9. Spalding D B 'A General Purpose Computer Program for Multi-Dimensional One- and Two-Phase Flow' Mathematics and Computers in Simulation, 23, 267, 1981

10. Bos W G, Vanden Elsen T, Hoogendoorn C J and Test F L 'Numerical Study of Stratification of a Smoke Layer in a Corridor Comb Science and Technology 38, 227, 1984.

11. Cox $G$ and Kumar $S$ 'Field Modelling of Fire in Forced Ventilated Enclosures, Combustion Science and Technology* 52, 7, 1987.

12. Markatos $N$ C, Malin $M \quad R$ and Tatchell D G 'Computer Analysis of Three-Dimensional Turbulent Flows Around Ships Hulls' Proc Inst Mech Eng 194, 239, 1980.

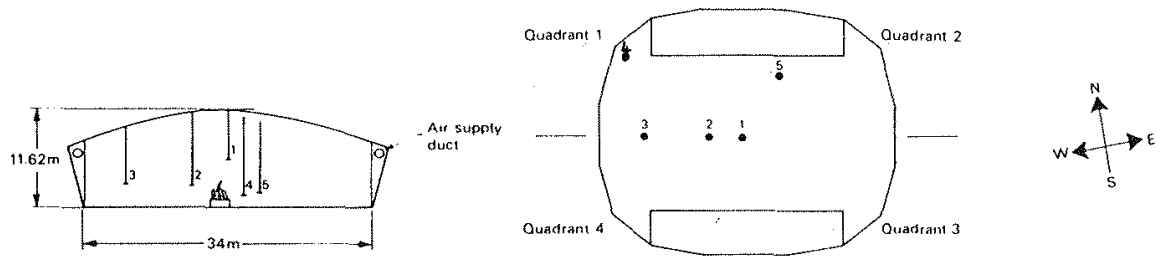

Fig 1: Experimental Dome Showing Thermocouple Columns 1-5

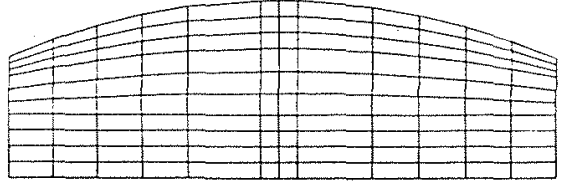

Body-Fitted

Fig 2: Numerical Grids Used in Computations

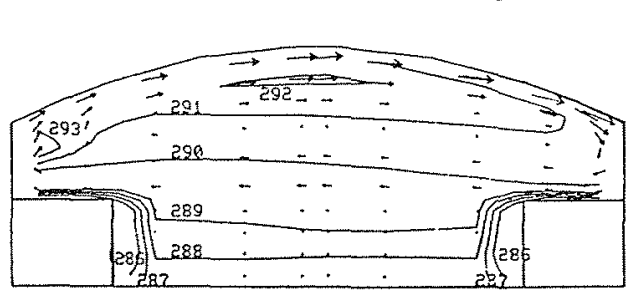

Fig 3: Pre-Fire Conditions

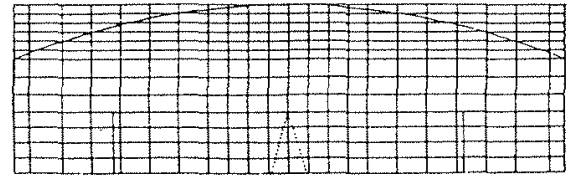

Cylindrical Polar

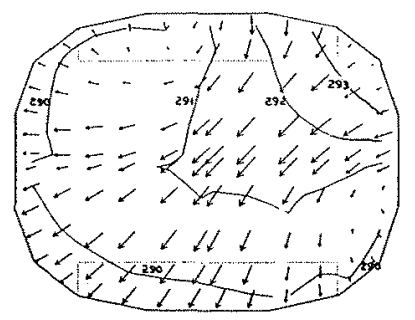




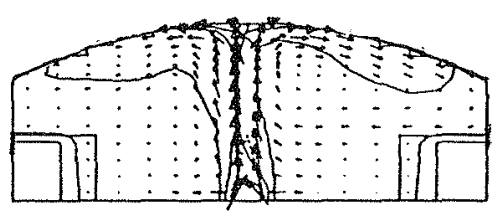

$\frac{1}{2} m$

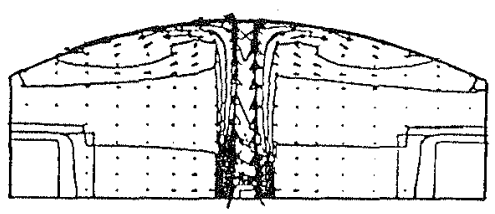

$2 m$

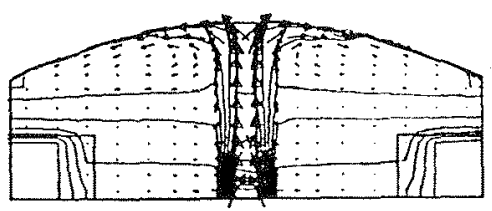

$6 \mathrm{~m}$

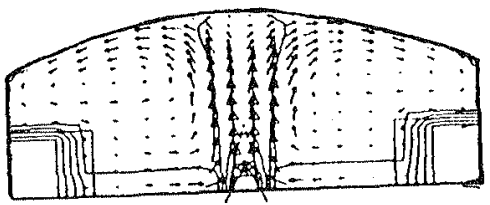

$10 \mathrm{~m}$

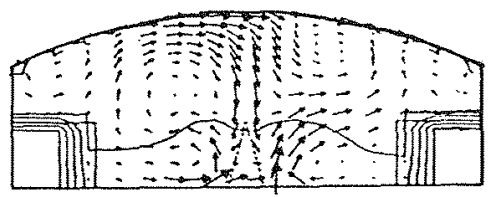

$11 \frac{1}{2} m$

Fig 4: Temperature Contours and Velocities During Fire and Post Fire Stages Viewed from East

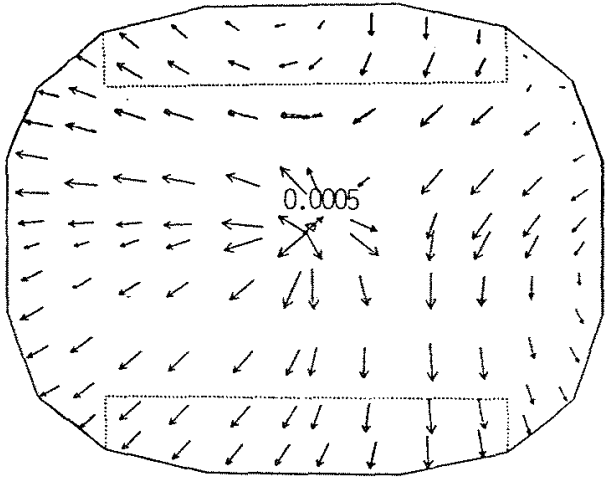

$14 \mathrm{~s}$
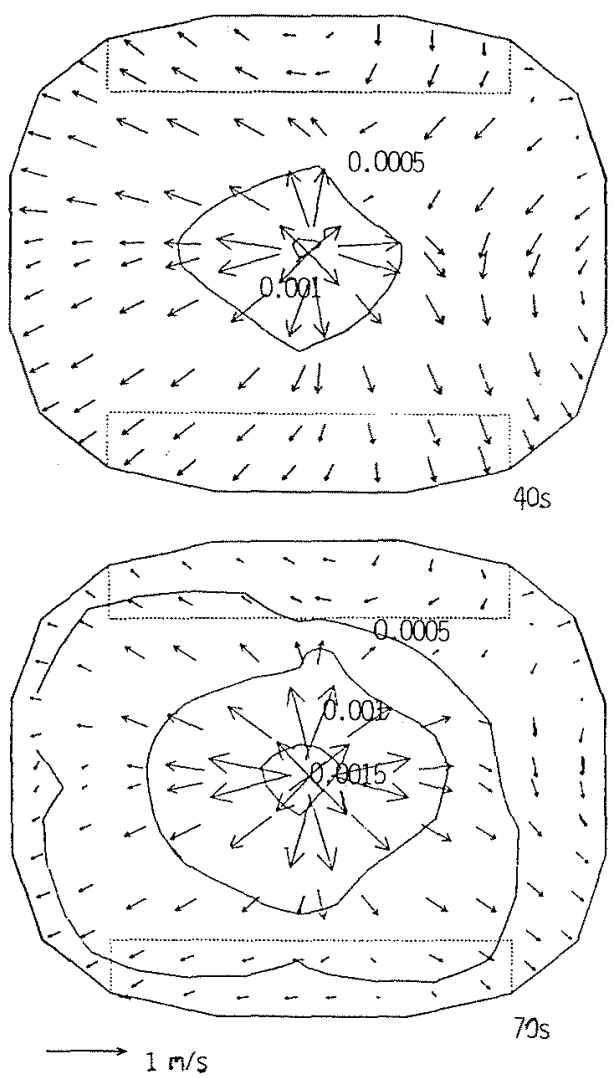

Fig 5: Velocity and Fuel Mixture Fraction. Contours Under the Ceiling in the Growth Phase of the Fire 


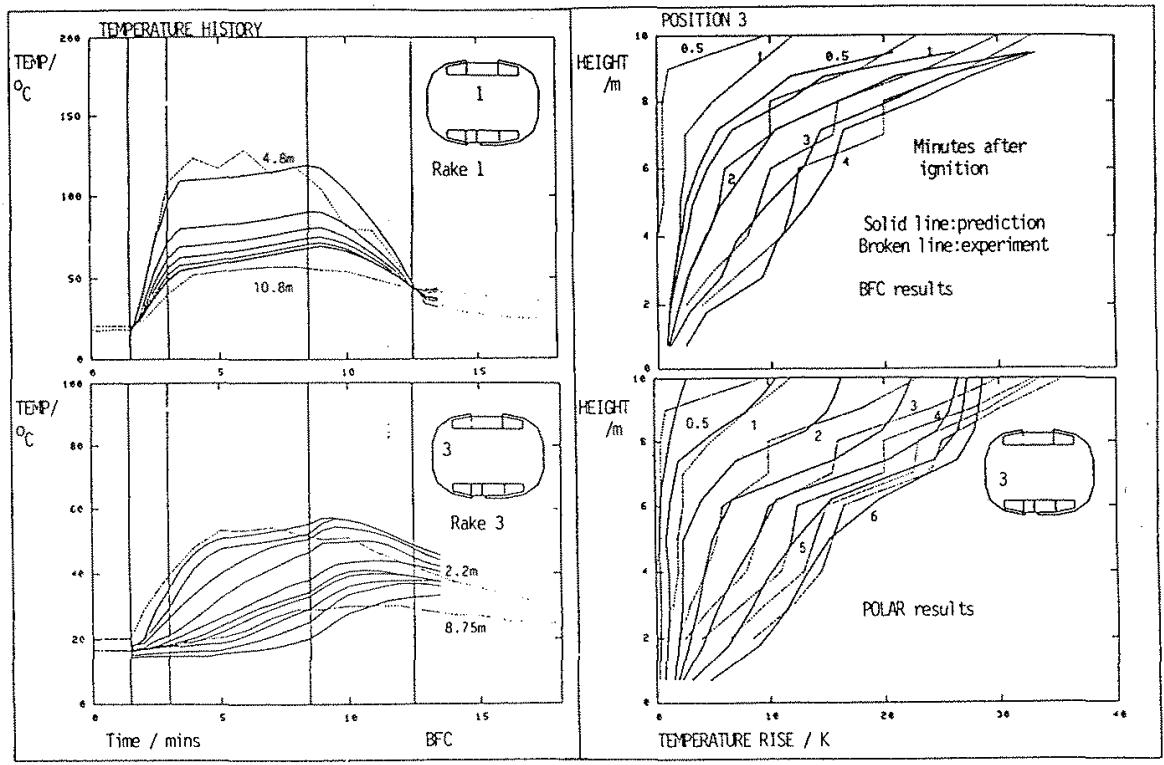

Dotted lines represent limits of experimental data

Fig 6: Predicted and Measured Temperature Histories at Stations 1 and 3 with the bfc Grid
Fig 7: Temperature Rise Predicted by Both Grids and Measured at Station 3

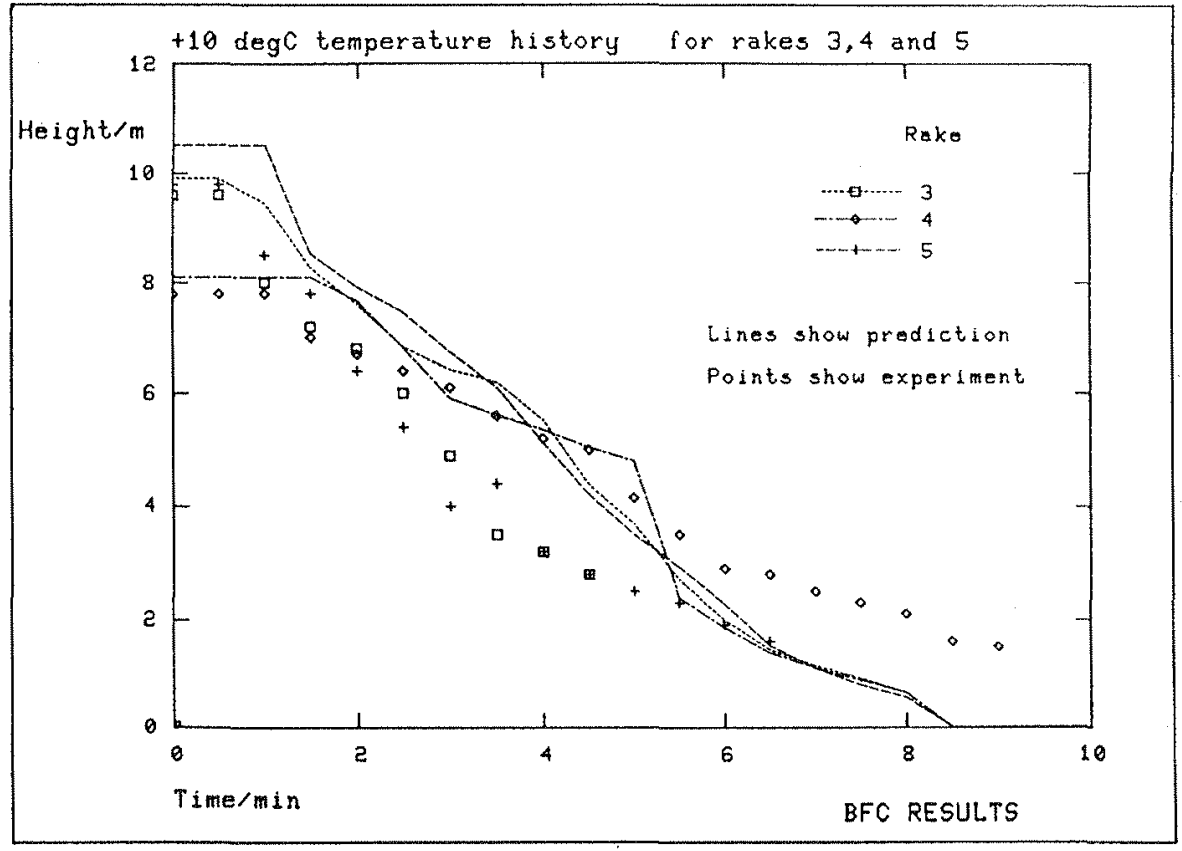

Fig 8: The Height of Ten Degree Rise Contour as a Function of Time are Rakes 3,4 and 5 